\title{
Automation of Angular Reconstitution for Facilitating the Construction of Initial Models in Single-Particle Electron Microscopy
}

\author{
Dmitry Lyumkis*, Sunita Nayak*, Ronald R. Milligan*, Clinton S. Potter*, Bridget Carragher* \\ * National Resource for Automated Molecular Microscopy and Department of Cell Biology, \\ The Scripps Research Institute, La Jolla, CA, 92037
}

Structure determination of a novel macromolecular complex via single particle electron microscopy depends upon overcoming the fundamental challenge of assigning the correct orientational Euler angles to 2-D projections, so as to obtain a reliable 3-D reconstruction. By establishing the relationship between consecutive 2-D projections based on "common line" similarity criteria, the angular reconstitution method assigns Euler angles without the requirement of physical specimen tilting, and is therefore capable of producing 3-D reconstructions directly from the data at hand [1]. Since its introduction, this ab initio approach has become one of the primary tools for constructing initial models, but its general reliance on manual user input leads to the recognized difficulty of reconstructing reliable volumes, particularly ones without symmetry. In a study that illustrated the effects of different reconstruction techniques, Cheng et al. noted that angular reconstitution would output different results and depended upon the order in which images were introduced into the reconstruction procedure [2]. We visually demonstrate this issue with the Transferrin-Transferrin Receptor (Tf-TfR) data used in their study, as well as the 70S ribosome. The main problem at hand is that all resulting reconstructions exhibit an extreme inter-volume heterogeneity, even when featured and well-aligned class averages are used as input.

To address this drawback a highly automated and iterative approach has been developed, the input to which consists only of a set of well-aligned class averages and an optional raw particle stack for refinement. The overall scheme involves five steps: initially, (1) a similarity matrix between input class averages is generated for a correlation-based assessment of a sequence of addition of 2-D images into angular reconstitution; (2) the latter is used to construct $\mathrm{N}$ different models (N specified by the user), which are (3) aligned into a single orientation by a 3-D maximum-likelihood approach [3] and (4) grouped into 3-D class averages by a novel method of clustering with affinity propagation [4]; (5) the most consistent model is chosen from several statistical analyses and may be optionally used as input for an iterative refinement against a raw-particle stack. We demonstrate the method's wide applicability and ease of use with five real datasets, comparing initial models and refinements of the $70 \mathrm{~S}$ ribosome, the $50 \mathrm{~S}$ ribosomal subunit, Transferrin-Transferrin Receptor (Tf-TfR), Dicer-TRBP, and Cop II cages. In doing so, we wish to make the point that the prevailing issues plaguing attempts to create a reliable initial model through the well-established angular reconstitution approach can be reduced or even eliminated by implementing our procedure [5].

\section{References}

[1] van Heel, M. Angular reconstitution: a posteriori assignment of projection directions for 3D reconstruction. Ultramicroscopy 21 (1987) 111-124. 
[2] Cheng, Y., et al. Single Particle Reconstructions of the Transferrin-Transferrin Receptor Complex Obtained with Different Specimen Preparation Techniques. Journal of Molecular Biology 355 (2006) 1048-1065.

[3] Scheres, Sjors et al. Averaging of electron subtomograms and random conical tilt reconstructions through likelihood optimization. Structure 17 (2009) 1563-1572.

[4] Frey, Brendan J. and Dueck, Delbert. Clustering by passing messages between data points. Science 315 (2007) 972-976.

[5] This research was conducted at the National Resource for Automated Molecular Microscopy (NRAMM) and funded by grants from the National Institute of Health (NIH) through the National Center for Research Resources (NCRR) P41 program (Grants RR17573 and RR023093).

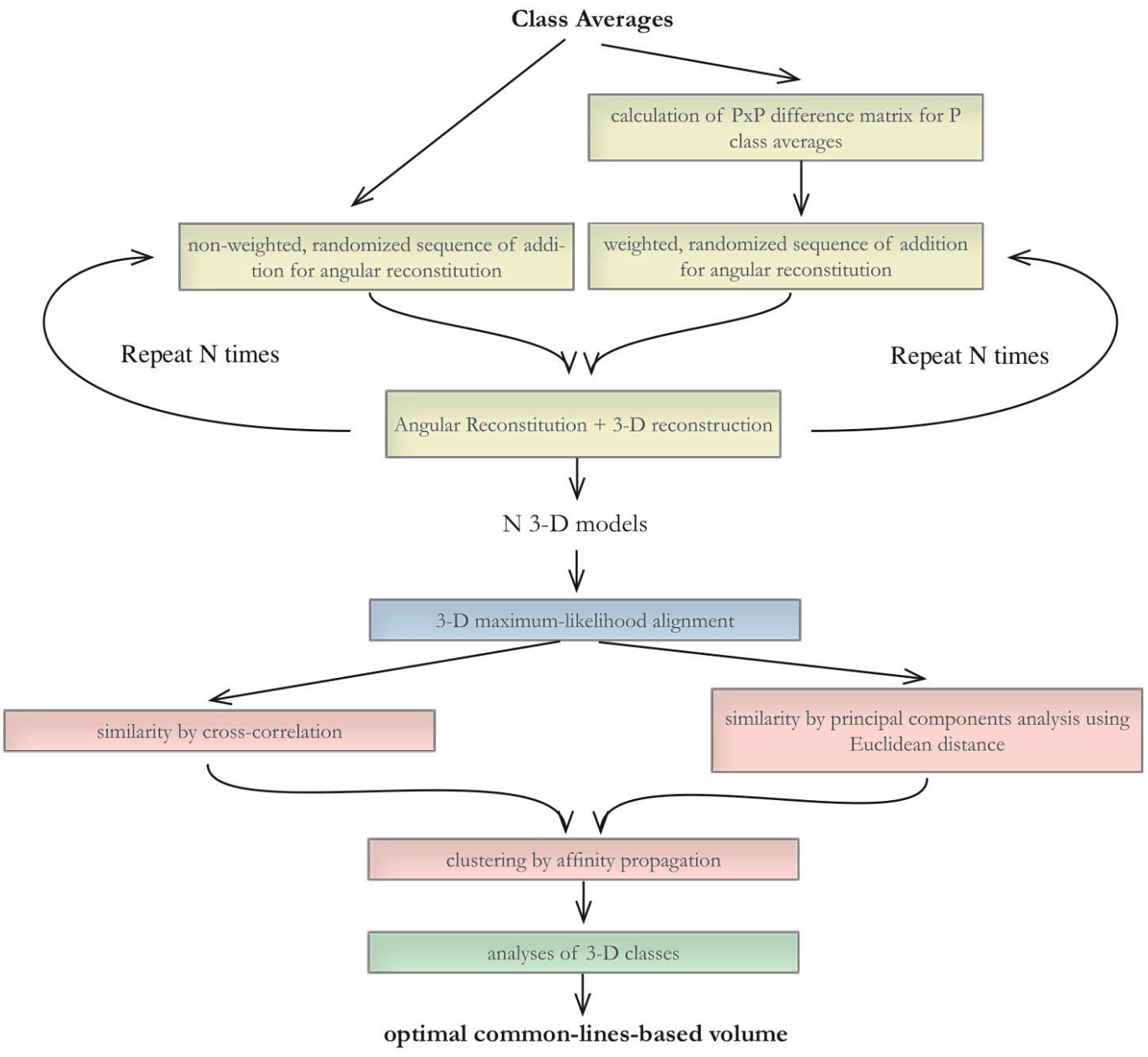

FIG 1: The overall scheme of automated angular reconstitution. Yellow colors refer to steps pertaining to the calculation of Euler angles and subsequent iterative 3-D reconstructions. Blue refers to 3-D alignment. Red refers to the steps pertaining to 3-D classification. Green refers to the selection of an optimal volume. 